\title{
A Novel Application of Extended Kalman Filter for Efficient Information Processing In Subsurfaces
}

\author{
Dimple Juneja \\ MM Institute of Computer \\ Technology \& Business \\ Management, MM University, \\ Mullana (Ambala)
}

\author{
Atul Sharma \\ MM Institute of Computer \\ Technology \& Business \\ Management, MM University, \\ Mullana (Ambala), \\ Haryana, India
}

\author{
A.K Sharma \\ Department of Computer \\ Science and Engineering \\ YMCA University of Science \& \\ Technology, Faridabad, \\ Haryana, India
}

\begin{abstract}
Recent works indicates that innovative deployment of sensors in subsurfaces can beneficially support the production of oil and gas. The data which is sensed by such sensors is usually corrupted with noise. Filtering is desirable in such embedded systems in order to smooth out such fluctuations that otherwise would shorten the lifespan of sensors. This contribution presents a unique application of Kalman filtering technique for processing such sensitive information because sensor readings are usually imprecise due to strong variations in environment and also, computation has to be much more energy efficient than communication. Out of the various filtering algorithms available, we have chosen to apply Kalman filter, primarily because it works well both in theory and practice and moreover, it is able to minimize the variance of estimation error i.e. filters noise from the actual signal more accurately.
\end{abstract}

Keywords: Wireless Sensor Networks, Kalman Filtering Algorithm, Extended Kalman Filter, Information Processing, Estimation Error.

\section{INTRODUCTION}

In the past few years, innovative oil monitoring techniques $[4,16,21,22,25,26]$ deployed in oil wells or sub-surfaces have proved to be an efficient way of measuring various parameters such as pressure, temperature, volume of oil available at various locations. Such innovative systems comprise of thousands of heterogeneous sensors which continually sense the environment. Since, the sensors are constrained in terms of energy, therefore, in order to be efficiently benefited from such systems, information that is actually promising shall only be routed so as to avoid useless energy drain and hence shorten the lifespan of WSN [9,11]. In order to meet, the above stated objective, one must employ the technique of collaborative signal processing $[2,8]$ and filter the unnecessary noise locally. Collaborative signal processing leads to information fusion which in turn reduces the data volume to be routed. However, the authors feel that this fused information must be processed i.e. filtered before routing because sensors readings are usually imprecise due to strong variations in the environment and hence information is corrupted with noise. In order to filter the sensed data, tools known as Kalman filters [23] can be used as these are often being used in embedded control systems for estimating the accurate range of process variables. This paper is structured into four sections. Section 1 provides a brief introduction of this work. Section 2 provides an overview of Kalman filters and the challenges in information processing. Section 3 presents our contribution and finally section 4 concludes with future work.

\section{PRELIMINARIES AND RELATED WORK}

The section provides a broad overview of Kalman Filter and design challenges in Information processing in WSN.

\subsection{Kalman Filters}

The Kalman filter is a mathematical model which describes equations to estimate the state of a process in a recursive computational manner [24]. The filtering algorithm is highly efficient for minimizing the mean of squared error, estimations of past, present and future states. The purpose of this section is to provide an insight to implementation of Kalman Filtering for Information Processing within Wireless Sensor Networks deployed in Sub-Surface.

In order to apply Kalman filter, the process to be measured shall be such that it can be described as linear system. A linear system can be expressed by a difference equation and a measurement equation as given by eq.(1) and eq.(2) respectively.

$$
\begin{gathered}
x_{k+1}=A x_{k}+B u_{k}+w_{k} \\
z_{k}=H x_{k+1}+v_{k}
\end{gathered}
$$

In the above equations,

$x$ : the state of the system,

$A: n \times n$ matrix relates the state at the previous time step $\mathrm{k}-1$ to the state at the current step $\mathrm{k}$, in the absence of either a driving function or process noise. Note that in practice, A, might change with each time step, but here we assume it is constant.

$B: n \times l$ matrix relates the optional control input $\mathrm{u}$ to the state $\mathrm{x}$.

$H: m \times n$ matrix in the measurement equation relates the state to the measurement $\mathrm{zk}$. In practice, $\mathrm{H}$ might change with each time step or measurement, but here we assume it is constant.
$k:$ the time index;
2: is a known input to the system;
$z:$ is the measured output; and 
$w$ : the process noise, and

$v$ : the measurement noise.

Given these two equations, we measure $z$, which is a function of $x$ that is corrupted by the noise $v$ as we cannot measure

$x$ directly. It shall be noted that $z$ can only be used to obtain an estimate of $x$, however, the information in $z$ is also corrupted by noise. Also, the above two equations indicates that Kalman Filter is recursive in nature i.e. it primarily estimates the state of the process at some arbitrary time and the feedback in the form of noise is observed.

It is evident that using Kalman filter, one is able to estimate the state of a discrete-time controlled process that is governed by a linear stochastic difference equation. However, an interesting application of the mentioned filter would be in case of non-linear processes i.e. the measurement relationship is non-linear. This is where Extended Kalman Filter (EKF)[24] comes in. Let us assume that the process under consideration is a non-linear process and for a state vector $x$, the process is now governed by the two non-linear stochastic difference equations given in (3) and (4) known as time-update and measurement-update equations respectively.

$$
\begin{aligned}
& \hat{x}_{k+1}=f\left(\hat{x}_{k}, u_{k}, w_{k}\right) \\
& \hat{z}_{k+1}=q\left(\hat{x}_{k+1}, v_{k}\right)
\end{aligned}
$$

where, the random variables $w$ and $v$ again represent the process and measurement noise. $f$ and $q$ are two non-linear functions where former relates the state at the previous time step to the state at the current time step and later relates the state $x_{k}$ to the measurement $z_{k}$. Here, matrices A, B and $\mathrm{H}$ (as mentioned in eq.(1)) are partial derivatives of functions $f$ and $q$ respectively. In practice of course one does not know the individual values of the noise $w_{k}$ and $v_{k}$ at each time step. However, one can approximate the state and measurement vector without them as given in eq.(5) and eq.(6) respectively.

$$
\begin{aligned}
& \hat{x}_{k+1}=f\left(\hat{x}_{k}, u_{k}, 0\right) \\
& \hat{z}_{k+1}=q\left(\hat{x}_{k+1}, 0\right)
\end{aligned}
$$

where, $\hat{x}_{k+1}$ is some a posteriori estimate of the state (from a previous time step k) [24].

Since, measurement relationship of parameters such as pressure, temperature, volume of oil in a particular vicinity etc., in oil well is a non-linear, this work aims to apply an EKF that linearize the estimation around the current estimate using the partial derivatives of the process and measurement functions to compute estimates even in the face of non-linear relationships.

\subsection{Design Challenges in Information Processing}

Information processing controls the process of transforming raw data collected via sensor readings, into useful and meaningful information to be used by scientists and researchers. Since beginning, due to energy constraints, Information processing has been active research area in wireless sensor networks $[8,10]$. Current research efforts include system state estimation [1], sensor management [9], collaborative signal processing [20], information fusion [12], information filtering, distributed compression[13], sensor querying and tasking [14], mobile-agent based information processing [10], and distributed inference and learning[17, $18,19]$ in wireless sensor networks just to name a few.

Transmission of packets over wireless media is always a challenging task $[3,6]$ in WSN due to resource scarcity of sensor network. At the same time, data transmission always remains the highest energy consumption factor in any network, and hence needs to be controlled efficiently. The best way available for saving energy and hence increasing lifetime of networks is to implement efficient data gathering as well as information processing techniques, so that only the minimal amount of data is transmitted over the wireless media.

Design challenges of the information processing in a wireless sensor network, includes data extraction, representation, data manipulation and propagation of refined information [7]. It aims at providing robust and efficient techniques to process high volume of data generated from large number of sensors, while remaining modest on available resource consumption. Some of biggest challenges of information processing includes, and are not restricted to data sampling, data storage, data aggregation, data fusion, data query and retrieval. Other challenges include identification of multiple concurrent physical phenomena [12] leading to duplicate data values, estimation of state, information compression and replication.

The main challenges involved in information processing design include the organization of participating sensor nodes into various clusters, identity identification of multiple physical attributes (viz. temperature, pressure, velocity etc), estimation of these physical attributes, and aggregation / processing and transmission of estimated results.

An in-depth evaluation of the above section indicates that researchers have been putting efforts to meet the challenges in information processing. Although, Information processing in WSN, has been an active area of research, but the literature indicates that researchers have remained silent towards the application of Kalman filter in this domain, especially in Subsurfaces where sensors are being deployed for monitoring. The upcoming section aims to address this unaddressed issue.

\section{THE PROPOSED WORK}

The section focuses upon the applying EKF for Information Processing in WSN in sub-surfaces and in non-deterministic environments, in general. Our previous works $[15,16]$ proposed how innovative deployment of sensors can beneficially support the production of oil and gas. Further, a query driven routing protocol [14] also addressed the issue pertaining to routing of information in such non-deterministic environments. One of the primary assumptions in these works are heterogeneous nodes i.e. to measure the modalities present within subsurface, various kind of sensor nodes such as temperature sensor, pressure nodes, acoustics nodes, flow nodes etc. are taken into consideration.

For the efficient production of heavy crude, the requirement is the continuous monitoring of all pertinent parameters throughout the production process. The parameters which are routinely measured are: annulus pressure, well head pressure, temperature, Oil Volume and flow rate. However, to the author's knowledge, a suitable calculation method or formula has not been published except for a few empirical formulae which are suitable only over a limited range. 
We had proposed to attach sensors to the oil to provide continuous liquid production rates. This system also allows to detect hazardous substances such as $\mathrm{H} 2 \mathrm{~S}$ or to connect sensors for monitoring of critical temperatures such as Boiling point temperatures. In order to avoid complexity of calculations, we would limit our scope to process pressure and Oil volume only, rather than processing all stated parameters. Assuming that in standard conditions, pressure $(p)$ at any depth $(h)$ at time $k$ can be computed (by using Pressure Sensor) as given by eq.(7)

$$
p_{k}=h_{k} \rho g
$$

where, $h=$ Depth of area,

$$
\begin{aligned}
& \rho=\text { density of oil, constant } \mathrm{kg} / \mathrm{m} 3 \\
& g=\text { acceleration due to gravity, } 9.8 \mathrm{~m} / \mathrm{sec} 2
\end{aligned}
$$

But the previous equation, does not give a precise value for $p_{k}$. Instead, the pressure will be perturbed by noise due to changing depth and other unfortunate realities. The pressure noise is a random variable that changes with position/depth. So a more realistic equation for $p$ would be as given by eq.(8):

$$
p_{k}=h_{k} \rho g+\tilde{u}
$$

where, $u_{k}=h_{k} \rho g^{\sim}$ is the noise in pressure depending upon the value of $h$ which may also be corrupted. Similarly, Oil Volumes (OV) in standard conditions is computed in accordance to eq.(9)

$$
V_{s c}=V_{s} \times S C F \times(1-S H R) \times(1-B S W) \times T
$$

Where, $\quad V_{s c}=$ Oil Volume at standard conditions

$V_{s}=$ Oil volumes measured by sensors critical

$S C F=$ Sensor Correction factor $=$ True volume/ Measured volume

$S H F=$ Shrinkage Factor

$B S W=$ Water and Sediment $\%$ measured

$T=$ Temperature volume correction factor at standard conditions. Here, the temperature used to calculate the volume correction factor to standard conditions is the well temperature measured using standard temperature sensors.

As per eq.(8), a similar expression for oil volume can be given as in eq(10).

$$
V_{k+1}=V_{s c}+w_{k}^{\sim}
$$

where, $w_{k}=V_{s c}^{\sim}$ is the noise in the volume of oil measured. Now, we can define a state vector $\mathrm{x}$ (given by eq.(11)) that consists of pressure and volume of oil as

$$
x_{k+1}=f\left(p_{k+1}, V_{k+1}, h_{k+1}, u_{k}, w_{k}\right)
$$

and output vector is given by eq.(12).

$$
z_{k+1}=f\left(x_{k+1}, m_{k}^{\tilde{k}}\right)
$$

Here, in eq.(11) and eq.(12), $u_{k}, w_{k}^{\tilde{k}}$ are the process noise and $m_{k}$ is the measurement noise respectively. In order to estimate the Pressure $\mathrm{p}$ and Volume V, we need to estimate $x$ and $z$, hence the need of EKF. Clearly, the requirement is twofold: First, the estimated value should be same as expected value of state and second, measurement update should be accurate i.e. $z$ should be estimated with smallest possible error. Now, in order to have the true estimates of the desired parameters, we have to assume that the average value of the stated noise variables should be zero. Further, it is evident that $u_{k}, w_{k}$ and $m_{k}$ are independent random variables. Then the Pressure Noise Covariance and Oil Volume Noise Covariance matrices are defined as in eq.(13) and eq.(14) respectively.

$$
\begin{array}{r}
S_{P}=\mathrm{E}\left(u_{k} u_{k}^{T}\right) \\
S_{V}=\mathrm{E}\left(w_{k} \tilde{w}_{k}^{T}\right)
\end{array}
$$

Similarly, Measurement noise covariance is computed as given in eq.(15):

$$
S_{m}=\mathrm{E}\left(\tilde{m}_{k}^{m_{k}} \tilde{\sim}^{T}\right)
$$

where, $u_{k}^{\sim}, w_{k}^{\sim}$ and $m_{k}^{\sim T}$ are the transpose of respective noise parameters and $\mathrm{E}$ is the expected value. On the basis of these arguments, equations for EKF can be formulated as follows:

$$
\hat{x}_{k+1}=f\left(\hat{x}_{k}, u_{k}, 0\right)
$$

$$
\begin{gathered}
P_{k+1}=A_{k+1} P_{k} A_{k+1}^{T}+W_{k+1} S_{p} W_{k+1}^{T}+W_{k+1} S_{V} W_{k+1}^{T} \\
K_{k+1}=P_{k+1} H_{k+1}^{T}\left(H_{k+1} P_{k+1} H_{k+1}^{T}+S_{m}\right)^{-1} \\
\widehat{x}_{k+1}=K_{k+1}\left(z_{k+1}-q\left(\widehat{x}_{k+1}, 0\right)\right) \\
P_{k+1}=\left(1-K_{k} H_{k}\right) P_{k}
\end{gathered}
$$

Eq.(16) and (17) represent time update equations while eq.(18) to eq.(20) are measurement update equations. The time update equations compute the state and covariance estimates. $A$ and $W$ are the process Jacobian matrices and are computed as partial derivatives of $f$. The state and covariance estimates are corrected with measurement $z_{k+1}$. $K$ is the Kalman gain and $H$ is the measurement Jacobian at step $\mathrm{k}+1$. It shall be noted that Jacobian $H$ magnifies only the relevant component of the measured parameter. It further, demands that there is one to one mapping between the state and the measurement at some of the instances, else the process is treated as unobservable and filter diverges. It is intuitive from eq.(18) that if measurement noise i.e. $S_{m}$ is large, K will be small and the measurement $z_{k+1}$ would not be of much importance in computing $\hat{x}_{k+1}$, the next state and viceversa. Similar arguments may be applied to compute other pertinent parameters. Next subsection presents the 
implementation of the above work for estimating pressure and associated noise. Representing the computation of all parameters is out of scope of this paper.

\subsection{Implementation of the Proposed Extended Kalman Filter for Pressure Estimation}

Let us assume that pressure measurement in an oil well system is done by measurement of the depth $h$ at which the probe reaches at a time $t$ and hence,

$$
p(t)=\rho g h(t)
$$

If the probe starts from rest with a constant acceleration $a(=2$ $\mathrm{m} / \mathrm{s} 2$ ), the actual depth and probe velocity at $k+1$-th time step maybe expressed as:

$$
\begin{gathered}
h_{k+1}=h_{k}+u \Delta t+\frac{1}{2} a(\Delta t)^{2}+z_{h} \\
u_{k+1}=h_{k}+a \Delta t+z_{u}
\end{gathered}
$$

Where $z_{h}$ and $z_{u}$ are the process noises associated with depth and velocity respectively. The measured pressure is evaluated as:

$$
p_{k+1}=\rho g\left(h_{k+1}+v\right)
$$

Where $v(=5 \mathrm{~m}$, one standard deviation $)$ is the noise associated with the measurement of depth, $\rho=800 \mathrm{~kg} / \mathrm{m} 3$ and $g=10 \mathrm{~m} / \mathrm{s} 2$. Assuming the acceleration noise to be $0.1 \mathrm{~m} / \mathrm{s} 2$, the Kalman algorithm is executed in order to filter the noise from the measured pressure signal and obtain the estimated pressure signal as shown in Figure 1. The errors in the measured and estimated pressures are shown in Figure 2. It is clear that Kalman filter greatly reduces the measurement noise. Similar, simulations can be performed for any other parameter observed by sensor nodes.

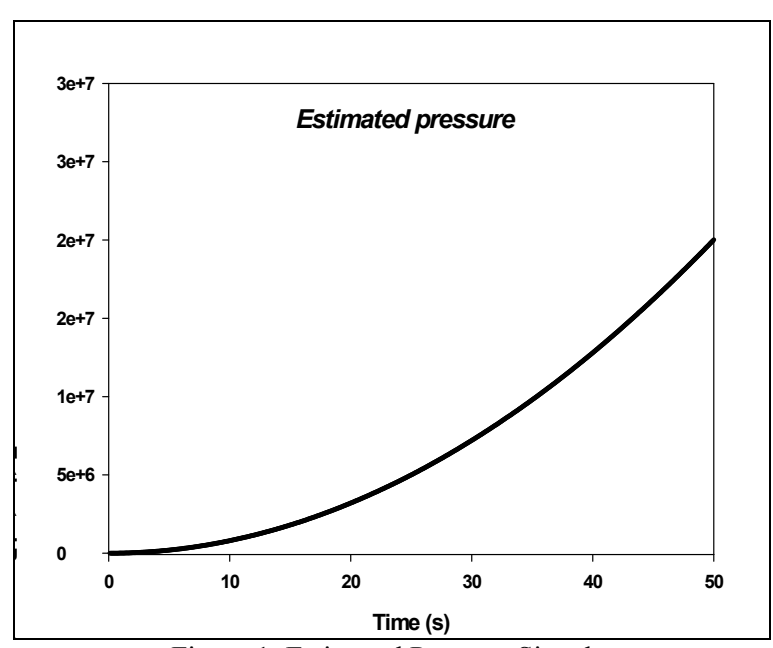

Figure 1: Estimated Pressure Signal

\section{CONCLUSIONS AND FUTURE WORK}

The application of this new technology and information processing techniques could lead to constant and effective monitoring of oil wells. This work proposed a novel method for critical measurements which are not only accurate but also filtered leading to better diagnosis, monitoring of oil wells and a more efficient use of sensor energy. The electronically acquired data can be distributed to the scientists for better analysis and also gives an opportunity to view the data in real time. On the other side, some sensors are prone to drift and it is important to perform post job calibrations to confirm the accuracy of the data. Although, the work is still in its infancy stage, however we have made an attempt to propose a novel information processing strategy using EKF in Oil Wells which makes this work unique.

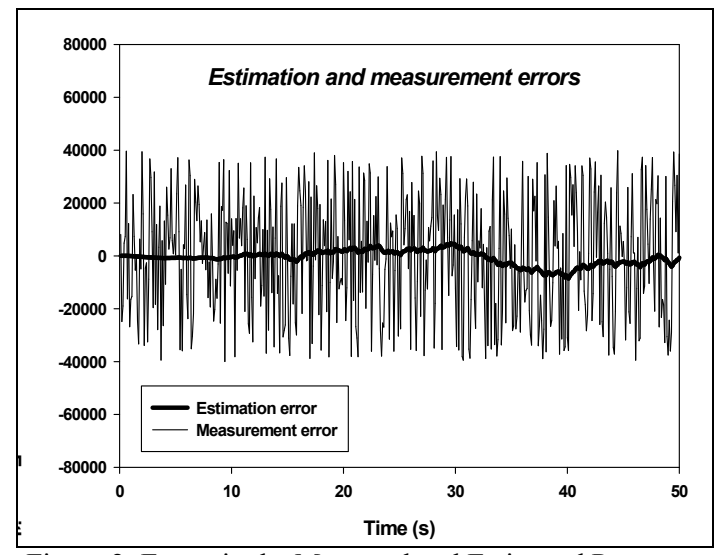

Figure 2: Errors in the Measured and Estimated Pressures

\section{REFERENCES}

[1] A.D'Costa, V.Ramachandran and A.M.Sayeed, "Distributed Classification of Gaussian Space-Time Sources in Wireless Sensor Networks", IEEE Journal on Selected Areas in Communications, Vol. 22, No. 6, August 2004.

[2] A.D'Costa and A.M.Sayeed, "Collaborative Signal Processing for Distributed Classification in Sensor Networks", In the Second International Workshop on Information Processing in Sensor Networks (IPSN '03), Palo Alto, CA, April, 2003.

[3] B.Chen, L.Tong and P.K.Varshney, "Channel-Aware Distributed Detection in Wireless Sensor Networks", IEEE Signal Processing Magazine, Vol. 23, No. 4, July 2006.

[4] C. A. Gonclaves, P. K. Harvey, and M. A. Lovell, "Prediction Of Petrophysical Parameter Logs Using A Multilayer Backpropagation Neural Network," in Developments in Petrophysics: Geological Society Special Publication. London, U.K.: Geological Society, 1997, pp. 169-180

[5] D.Guo and X.Wang, "Dynamic Sensor Collaboration via Sequential Monte Carlo", IEEE Journal on Selected Areas in Communications, Vol. 22, No. 6, August 2004.

[6] D.Li, K.Wong, Y.H.Hu and A.Sayeed, "Detection, Classification and Tracking of Targets", IEEE Signal Processing Magazine, March 2002. 
[7] F.Zhao and J.Shin and J.Reich, "Information-Driven Dynamic Sensor Collaboration for Tracking Applications", IEEE Signal Processing Magazine, March 2002.

[8] F.Zhao, J.Liu; J.Liu, L.Guibas and J.Reich, "Collaborative Signal and Information Processing: an Information-Directed Approach", Proceedings of IEEE, Vol. 91, No. 8, August 2003.

[9] G. Pottie, W. Kaiser. "Wireless Integrated Network Sensors." Communications of the ACM, 43(5):51 - 58, May 2000.

[10] H.Qi, Y.Xu and X.Wang, "Mobile-Agent-Based Collaborative Signal and Information Processing in Sensor Networks", Proceedings of the IEEE, Vol. 91, No. 8, August 2003.

[11] I.F.Akyildiz, W.Su, Y.Sankarasubramaniam and E.Cayirci, "A Survey on Sensor Networks", IEEE Communications Magazine, August 2002.

[12] J.H.Kotecha, V.Ramachandran and A.M. Sayeed, "Distributed Multitarget Classification in Wireless Sensor Networks", IEEE Journal on Selected Areas in Communications, Vol. 23, No. 4, April 2005.

[13] J.Xiao, A.Ribeiro, Z.Luo and G.B.Giannakis, "Distributed Compression-Estimation Using Wireless Sensor Networks", IEEE Signal Processing Magazine, Vol. 23, No. 4, July 2006.

[14] Juneja Dimple, Sharma Atul \& Sharma A.K., "A Query Driven Routing Protocol for Wireless Sensor Nodes in Subsurface". International Journal of Engineering Science and Technology. Vol. 2 No. 6, July 2010, pp. 1836-1843

[15] Juneja Dimple, Sharma Atul, Kumar Punit, Iyengar S.S, \& Sharma A.K, "A Novel and Efficient Algorithm for Deploying Mobile Sensors in Subsurface". Computer and Information Science. Vol 3, No 2 (2010), pp.94105 .

[16] Juneja Dimple, Sharma Atul \& Sharma A.K., "On The Role Of Wireless Sensor Networks In Subsurface Exploration: An Overview", 2009 IEEE International Advance Computing Conference (Iacc 2009) 3159-3162
[17] L.J.Guibas, "Sensing, Tracking, and Reasoning with Relations", IEEE Signal Processing Magazine, March 2000.

[18] M.Cetin, L.Chen, J.W. Fisher III, A.T. Ihler, R.L.Moses, M. J.Wainwright and A.S. Willsky, "Distributed Signal Processing in Sensor Networks", IEEE Signal Processing Magazine, Vol. 23, No. 4, July 2006.

[19] R.Brooks, P.Ramanathan and A.Sayeed, "Distributed Target Classification and Tracking in Sensor Networks",Proceedings of the IEEE, Vol. 91, No. 8, August 2003.

[20] W.Bajwa, J. Haupt, A.Sayeed and R.Nowak, "Joint Source-Channel Communication for Distributed Estimation in Sensor Networks", submitted to the IEEE Transactions on Information Theory, August 2006.

[21] Wilamowski Bogdan M, Kaynak Okyay," Oil Well Diagnosis by Sensing Terminal Characteristics of the Induction Motor", IEEE Transactions On Industrial Electronics, Vol. 47, No. 5, October 2000, 1100-1107.

[22] XIAO, ZU-QI, South Huang Hai Oil Corp, "The Calculation of Oil Temperature in a Well", 1987. An unpublished article submitted to Society of Petroleum Engineers http://www.onepetro.org/mslib/app/Preview.do?paperN umber $=00017125 \&$ societyCode $=$ SPE

[23] Dan Simon, “ Kalman Filtering” Embedded Systems Programming, June 2001, pp:72-79.

[24] Greg Welch , Gary Bishop, “ An Introduction to Kalman Filter" UNC-Chapel Hill,TR 95-041, July 24,2006, pp:1-16.

[25] Clayton M. Costa1, Cicília R. M. Leite2, Francisco M. M. Neto3, Pedro F. R. Neto, "AMSO: Real-Time System For Oil Wells Onshores Using Sensors Network" avaialble online at www.eatis.org/eatis2010/portal/paper/memoria/html/file s/39.pdf

[26] Pal Skalle, Agnar Aamodt, "Knowledge-Based Decision Support In Oil Well Drilling", Intelligent Information Processing II: IFIP International Conference on Intelligent Information Processing, pp:443-455 